\title{
INTERVENÇÕES DA TERAPIA OCUPACIONAL COM PACIENTES PÓS-FEBRE CHIKUNGUNYA: UM RELATO DE EXPERIÊNCIA
}

\author{
INTERVENCIONES DE TERAPIA OCUPACIONAL CON PACIENTES DESPUÉS DEL \\ FIEBRE CHIKUNGUNYA: UN INFORME DE EXPERIENCIA
}

OCCUPATIONAL THERAPY INTERVENTIONS WITH POST CHIKUNGUNYA FEVER PATIENTS: AN EXPERIENCE REPORT

\section{Lucas de Paiva Silva'; Beatriz do Nascimento Silva²; Angélica da Silva Tenório; Ana Karina Pessoa da Silva Cabral ${ }^{4}$; Danielle Carneiro de Menezes Sanguinetti ${ }^{5}$ y Daniela Salgado Amaral ${ }^{6}$}

1 Terapeuta Ocupacional graduado pela Universidade Federal de Pernambuco (UFPE). Pós-Graduando pelo Programa de Residência Multiprofissional em Reabilitação Física no Instituto de Medicina Integral Professor Fernando Figueira (IMIP). Instituição afiliada principal: Instituto de Medicina Integral Professor Fernando Figueira (IMIP). ResearchedID: https://publons.com/researcher/3808870/lucas-de-paiva-silva/. ORCID: https://orcid.org/oooo-ooo3-4258-6591. Endereço: Universidade Federal de Pernambuco, Departamento de Terapia Ocupacional, Av. Jorn. Aníbal Fernandes, 273, Cidade Universitária, CEP 50730-120, Recife - PE, Brasil. Correio eletrônico: lucaspaiva.to@gmail.com. Número de telefone: +55 (81) 987070084

2 Graduanda do curso de Terapia Ocupacional pela Universidade Federal de Pernambuco (UFPE) Instituição afiliada principal: Universidade Federal de Pernambuco (UFPE). ResearchedID: https://publons.com/researcher/3808888/beatriz-do-nascimento-silva/. ORCID: https:// orcid.org/oooo-ooo2-8950-3127. Endereço: Universidade Federal de Pernambuco, Departamento de Terapia Ocupacional, Av. Jorn. Aníbal Fernandes, 273, Cidade Universitária, CEP 50730-12O, Recife - PE, Brasil. Correio eletrônico: eubeanascimento@gmail.com. Número de telefone: +55 (81) 9372-6929.

3 Fisioterapeuta graduada pela Universidade Federal de Pernambuco (UFPE). Docente do Departamento de Fisioterapia da Universidade Federal de Pernambuco (UFPE). Doutora em Nutrição pela Universidade Federal de Pernambuco (UFPE), Mestre em Biofísica pela Universidade Federal de Pernambuco (UFPE). Instituição afiliada principal: Universidade Federal de Pernambuco (UFPE). ResearchedID: https://publons. com/researcher/3810325/angelica-da-silva-tenorio/. ORCID: https://orcid.org/oooo-ooo2-7066-9047. Endereço: Universidade Federal de Pernambuco, Departamento de Fisioterapia, Av. Jorn. Aníbal Fernandes, 173, Cidade Universitária, CEP 50740-560, Recife - PE, Brasil. Correio eletrônico: tenorioangelica@yahoo.com.br. Número de telefone: +55 (81) 9232-1442.

4 Terapeuta Ocupacional graduada pela Universidade Federal de Pernambuco (UFPE). Doutora em Design pela Universidade Federal de Pernambuco (UFPE), Mestre em Design pela Universidade Federal de Pernambuco (UFPE). Instituição afiliada principal: Docente do Departamento de Terapia Ocupacional da Universidade Federal de Pernambuco (uFPE) ResearchedID: https://publons.com/researcher/3827455/ ana-karina-pessoa-da-silva-cabral/ ORCID: https://orcid.org/oooo-ooo3-4693-7758. Endereço: Universidade Federal de Pernambuco, Departamento de Terapia Ocupacional, Av. Jorn. Aníbal Fernandes, 273, Cidade Universitária, CEP 50730-120, Recife - PE, Brasil. Correio eletrônico: anakarina.ufpe@gmail.com Número de telefone: +55 (81) 8848-6178.

5 Terapeuta Ocupacional graduada pela Universidade Federal de Pernambuco (UfPE). Doutora em Neuropsiquiatria e Ciências do Comportamento pela Universidade Federal de Pernambuco (UFPE), Mestre em Neuropsiquiatria e Ciências do Comportamento pela Universidade Federal de Pernambuco (UfPe). Docente do Departamento de Terapia Ocupacional da Universidade Federal de Pernambuco (ufPE) ResearchedID: https://publons.com/researcher/AAV-96o9-202O/. ORCID: https://orcid.org/oooo-0oo3-4894-749o. Endereço: Universidade Federal de Pernambuco, Departamento de Terapia Ocupacional, Av. Jorn. Aníbal Fernandes, 273, Cidade Universitária, CEP 50730-120, Recife - PE, Brasil. Correio eletrônico: danielle.sanguinetti@ufpe.br Número de telefone: +55 (81) 8292-1688.

6 Terapeuta Ocupacional graduada pela Universidade Federal de Pernambuco (UFPE). Doutoranda em Design e Expressão Gráfica pela Universidade Federal de Santa Catarina (UFSC), Mestre em Ciências da Saúde pela Universidade Federal de Pernambuco (UFPE). Docente do Departamento de Terapia Ocupacional da Universidade Federal de Pernamb.uco (UFPE) ResearchedID: https://publons.com/researcher/3810099/daniela-amaral. ORCID: https://orcid.org/oooo-ooo2-7227-4022. Endereço: Universidade Federal de Pernambuco, 


\title{
RESUMO
}

Introdução: Indivíduos acometidos pela Febre Chikungunya, a depender do grau de gravidade do quadro clínico, por muitas vezes, deixam de realizar algumas de suas atividades cotidianas devido a intensidade de dores. Deste modo, as perdas funcionais resultam em mudança nos papéis ocupacionais exercidos. Objetivos: Relatar a experiência da assistência terapêutica ocupacional com pacientes pós-Febre Chikungunya, e descrever as intervenções e estratégias utilizadas. Método: Estudo descritivo, do tipo relato de experiência a partir de vivências em projeto de extensão com 10 pacientes pós-Febre Chikungunya, realizado na clínica escola de Fisioterapia com colaboração do Departamento de Terapia Ocupacional da Universidade Federal de Pernambuco, durante o período de abril a dezembro de 2018. Resultados: Participaram do estudo 8 pacientes do sexo feminino e 2 do sexo masculino, com idade entre 48 e 74 anos. A Terapia Ocupacional colaborou com o projeto a partir de ações, como: avaliação terapêutica ocupacional, discussão em equipe, realização de grupo de autocuidado e de um grupo dialógico de discentes da Fisioterapia e Terapia Ocupacional, e um atendimento individualizado. Conclusão: As ações mostraram-se importantes para a construção do conhecimento sobre a doença e melhor enfrentamento da mesma a partir do foco da Terapia Ocupacional. Considerou-se que as intervenções terapêuticas ocupacionais podem ser individuais e/ou em grupo, utilizando-se como base os princípios de reumatologia sobre proteção articular e conservação de energia como importantes referenciais para o subsídio de ações terapêuticas.

\section{PALAVRAS-ChAVE}

Educação em Saúde; Equipamentos de Autoajuda; Febre de Chikungunya; Reumatologia; Terapia Ocupacional.

\section{RESUMEN}

Introducción: las personas afectadas por la fiebre chikungunya, según el grado de gravedad del cuadro clínico, a menudo no pueden realizar algunas de sus actividades diarias debido a la intensidad del dolor. De esta manera, las pérdidas funcionales resultan en cambios en los roles ocupacionales ejercidos. Objetivos: Informar la experiencia de asistencia de terapia ocupacional con pacientes después de la fiebre Chikungunya, y describir las intervenciones y estrategias utilizadas. Método: Estudio descriptivo, un informe de experiencia basado en experiencias en un proyecto de extensión con 10 pacientes después de la Fiebre Chikungunya, realizado en la clínica de la escuela de fisioterapia con la colaboración del Departamento de Terapia Ocupacional de la Universidad Federal de Pernambuco, durante el período de abril hasta diciembre de 2018. Resultados: el estudio incluyó a 8 pacientes de sexo femenino y 2 pacientes de sexo masculino, con edades comprendidas entre 48 y 74 años. La terapia ocupacional colaboró con el proyecto en base a acciones tales como: evaluación terapéutica ocupacional, discusión en equipo, grupo de autocuidado y grupo dialógico de estudiantes de fisioterapia y terapia ocupacional, y atención individualizada. Conclusión: Las acciones demostraron ser importantes para la construcción de conocimiento sobre la enfermedad y para enfrentarla mejor desde el enfoque de la Terapia Ocupacional. Se consideró que las intervenciones terapéuticas ocupacionales pueden ser individuales y/o grupales, basadas en los principios de la reumatología en la protección de las articulaciones y la conservación de la energía como referencias importantes para subsidiar acciones terapéuticas.

\section{PALABRAS ClaVE}

Educación para la salud; Equipo de autoayuda; Fiebre Chikungunya; Reumatología; Terapia ocupacional.

\begin{abstract}
Introduction: Individuals affected by Chikungunya Fever, depending on the severity of the clinical picture, often fail to perform some of their daily activities due to pain intensity. Thus, functional losses result in changes in occupational roles. Objectives: To report the experience of occupational therapeutic care with patients after Chikungunya Fever, and describe the interventions and strategies used. Methods: This is a descriptive, experience-based study from experiences in an extension project with 1o patients after Chikungunya Fever, conducted at the Physical Therapy School Clinic in collaboration with the Occupational Therapy Department of the Federal University of Pernambuco, from April to December from 2018. Results: Eight female and two male patients aged 48 to 74 years participated in the study. Occupational Therapy collaborated with the project through actions such as: occupational therapeutic evaluation, team discussion, self-care group and a dialogical
\end{abstract}

Departamento de Terapia Ocupacional, Av. Jorn. Aníbal Fernandes, 273, Cidade Universitária, CEP 50730-120, Recife - PE, Brasil. Correio eletrônico: danisamaral@hotmail.com. Número de telefone: +55(81)9606-8290. 
group of students of Physical Therapy and Occupational Therapy, as well as individualized monitoring by one of the project participants. Conclusion: The actions proved to be important for the construction of knowledge about the disease and better coping with it from the focus of Occupational Therapy. It was considered that occupational therapeutic interventions can be individual and/or group, based on the principles of rheumatology on joint protection and energy conservation as important references for subsidizing therapeutic actions.

\section{KEYWORDS}

Chikungunya Fever; Health Education; Occupational Therapy; Rheumatology; Self-Help Devices.

\section{INTRODUÇÃO}

O vírus Chikungunya é um alfavírus da família Togaviridae, causador de uma arbovirose e, consequentemente, um desafio para a população, pesquisadores e serviços de saúde, sendo identificado nas Américas no final do ano 2013. Entre os principais fatores favoráveis para a transmissão de doenças infecciosas, como a Febre Chikungunya (FC), ressalta-se a fácil proliferação e disseminação do vírus por meio de picada dos mosquitos Aedes aegypti e Aedes albopictus, mudanças climáticas e o crescimento do intercâmbio internacional. O vírus possui o período de incubação de dois a seis dias, e seus sintomas podem se manifestar de quatro a sete dias após a infecção. Em decorrência da até então ausência de vacina e medicação específica, todos os indivíduos se encontram susceptíveis a doença, o que caracteriza a Febre Chikungunya como atual desafio para a saúde pública brasileira (Donalisio e Freitas, 2015; Castro, Lima e Nascimento, 2016; Lima-Camara, 2016; Viana et al., 2018).

O primeiro caso de Febre Chikungunya registrado no Brasil ocorreu no estado do Amapá, tornando-se uma doença de difícil diagnóstico pela semelhança com outras arboviroses epidêmicas presentes no território brasileiro, como a Dengue e Zika (Aguiar et al., 2018). Diante do cenário endêmico, observou-se uma crescente busca da população afetada pelos serviços de saúde, enquanto os profissionais buscavam aprofundar os conhecimentos acerca da doença e suas particularidades, a fim de uma melhor resolutividade dos casos. Neste sentido, equipes de saúde incorporam métodos terapêuticos não farmacológicos, a citar a Terapia Ocupacional e a Fisioterapia, ao tratamento medicamentoso nos diferentes estágios e sintomas da doença aos pacientes acometidos pela FC (Viana et al., 2018; Silva et al., 2018).

A doença pode evoluir em três fases: aguda, subaguda e crônica. Na fase aguda, durante as três primeiras semanas, os indivíduos acometidos apresentam frequentemente febre alta, mialgia intensa, dores e edema simétricos principalmente nas articulações dos dedos, punhos, pés e tornozelos, além de náusea, fadiga e erupções cutâneas. A fase subaguda é marcada pela presença dos sintomas articulares como artralgia, artrite, mialgia, rigidez matinal que podem evoluir para a fase crônica diante das persistências dos sintomas por mais de três meses (Pineda et al., 2016; Marques et al., 2017).

A literatura aponta a artralgia, artrite e as condições reumatológicas como consequências frequentes da FC que mais comprometem a mobilidade e autonomia nos indivíduos acometidos, com destaque para a população idosa. Devido à possibilidade de cronificação, os sintomas da doença tendem a diminuir a qualidade de vida dos pacientes, uma vez afetando seu desempenho em atividades nas diferentes áreas ocupacionais (Azevedo, Oliveira e Vasconcelos, 2015; Castro, Lima e Nascimento, 2016; Viana et al., 2018).

Nesse sentido, Castro, Lima e Nascimento (2016) consideraram a Chikungunya como o vírus de maior relação com condições reumatológicas em indivíduos acometidos. Ainda segundo os autores, o vírus pode favorecer o desenvolvimento de uma doença reumática ou agravar o quadro sintomático de artrite previamente diagnosticada. Para Marques et al. (2017) o diagnóstico prévio de uma doença reumatológica indica a persistência dos 
sintomas articulares pós-Febre Chikungunya. Almeida et al. (2015) pontuam que tais limitações advindas de doenças reumatológicas afetam, além da feitura das atividades, os aspectos emocionais, as relações interpessoais e repercutem na qualidade de vida dessas pessoas.

Em relação ao comprometimento articular, o estudo de Marques et al. (2017) discute que essas queixas podem se apresentar como persistentes ou recidivantes. No que diz respeito ao tratamento, diante do fato da FC ainda não ter um medicamento específico ou vacina adequada, torna-se necessário esquemas medicamentosos para alívio dos sintomas. O repouso, a hidratação e as medicações antitérmicas são indicadas na fase aguda da doença na qual objetiva-se o alívio da dor musculoesquelética, enquanto os anti-inflamatórios não hormonais na fase subaguda. Já na fase crônica são recomendados os anti-inflamatórios e imunossupressores (Azevedo, Oliveira e Vasconcelos, 2015; Marques et al., 2017).

De acordo com as Recomendações da Sociedade Brasileira de Reumatologia para diagnóstico e tratamento da Febre Chikungunya, intervenções de reabilitação são recomendadas em todas as fases de evolução da doença, como medidas não farmacológicas complementares ao tratamento. A orientação e o estímulo à adoção de medidas preventivas do vetor transmissor do vírus Chikungunya tornam-se primordiais para impedir a disseminação dos mosquitos Aedes (Marques et al., 2017).

Orientações quanto a prática de exercícios individuais para as articulações, repousos, uso de órteses e adequação postural são algumas medidas que podem ser reforçadas para os pacientes pelos profissionais da saúde em uma abordagem contextualizada, preconizando a participação ativa dos sujeitos no processo de reabilitação. Deste modo, a educação em saúde é apontada como um potencial método na promoção de saúde dos indivíduos, tendo em vista o impacto do quadro clínico da Febre Chikungunya no desempenho dos indivíduos acometidos (Marques et al., 2017).

Nesse sentido, entendendo a ocupação humana como foco de suas intervenções, a Terapia Ocupacional é uma das profissões aptas a prestar assistência a pacientes infectados pelo vírus Chikungunya, tendo em vista que suas complicações podem implicar negativamente na realização de atividades em diferentes áreas ocupacionais, como atividades de vida diária (AVD), atividades instrumentais de vida diária (AIVD), participação social, lazer e trabalho. A depender do grau de gravidade de seu quadro clínico, indivíduos acometidos pela FC por muitas vezes deixam de realizar algumas de suas atividades cotidianas devido a intensidade de dores. Assim, suas perdas funcionais resultam em mudança nos papéis ocupacionais previamente exercidos (Parreira et al., 2013; Pineda et al., 2016).

Deste modo, posto que pacientes acometidos pela FC tendem a desenvolver ou agravar quadros reumatológicos, o terapeuta ocupacional pode assistir esta população com respaldo nos princípios da Terapia Ocupacional na reumatologia. Tais princípios englobam diferentes abordagens como a tecnologia assistiva e dispositivos de assistência, adaptações ambientais, educação em saúde individual ou em grupo, preconizando a participação ativa do indivíduo (Miranda, 2012).

Por meio de orientações, atividades e treino de habilidades, o terapeuta ocupacional pode auxiliar o indivíduo pós-FC considerando seus aspectos físicos, emocionais, cognitivos e sociais numa abordagem holística e centrada no paciente. O objetivo da intervenção é manter ou potencializar a capacidade funcional do público alvo em suas atividades significativas, além de manter a mobilidade articular, minimizar os desgastes articulares, para evitar deformidades (Miranda, 2012). Nesse contexto, os princípios da proteção articular e conservação de energia são comumente utilizados pelos terapeutas ocupacionais junto a pacientes com condições reumatológicas, uma vez que estes contemplam as especificidades no campo da reumatologia (Bianchin et al., 2010).

Diante do exposto, considerando a importância da assistência adequada e de qualidade ao indivíduo acometido pelo vírus Chikungunya, o objetivo deste artigo é relatar a experiência da assistência terapêutica ocupacional com pacientes pós-Febre Chikungunya e descrever as intervenções e estratégias utilizadas.

\section{MÉTodo}

Trata-se de um estudo descritivo, do tipo relato de experiência, a partir de vivências em projeto de extensão com pacientes pós-Febre Chikungunya (FC), realizado na clínica escola de Fisioterapia com colaboração do Departamento de Terapia Ocupacional da Universidade 
Federal de Pernambuco (UFPE), Recife/PE, Brasil, durante o período de abril a dezembro de 2018. A amostra do estudo foi composta por pacientes que estavam inscritos no projeto e em acompanhamento pela Fisioterapia, totalizando 10 indivíduos.

O projeto de extensão "Reabilitação Funcional Musculoesquelética pós-Febre Chikungunya" foi coordenado por uma docente do Departamento de Fisioterapia da UFPE, e teve a colaboração de docente e discentes do curso de graduação em Terapia Ocupacional da mesma instituição. O público-alvo do projeto foram pacientes com diagnóstico de FC que apresentassem manifestações musculoesqueléticas. A ação da Fisioterapia era realizada por meio da assistência baseada em condutas de cinesioterapia, recursos terapêuticos manuais e termoterapia, conforme as necessidades individuais verificadas nas avaliações cinético-funcionais. Os encontros aconteciam duas vezes por semana com duração de 2 horas cada, no período de 40 semanas no ano de 2018. O projeto também tinha como proposta unir as ações das áreas da Fisioterapia e da Terapia Ocupacional com estudantes destes cursos em atividades voltadas para o estudo e a intervenção dessas profissões em pacientes com diagnóstico de FC.

O instrumento utilizado pela Terapia Ocupacional na avaliação foi a Medida Canadense de Desempenho Ocupacional (СОРM), traduzido para língua portuguesa (Law et al., 2009), que abrange as atividades de autocuidado, produtividade e lazer e permite o levantamento das principais atividades realizadas com dificuldades pelo indivíduo, determinando o desempenho funcional e a satisfação (Souza et al., 2018). E nos casos de queixas ou comprometimentos funcionais em membros superiores, aplicou-se o Score for Assessment and quantification of Chronic Rheumatic Affections of the Hands (SACRAH), um instrumento validado para o Brasil (Ferreira e Marques, 2008). Este apresenta 23 itens que podem variar o a 10omm, onde o representa sem dificuldade para realizar as atividades, sem dor e sem rigidez nas mãos e 100 representa o maior grau de comprometimento para as mesmas categorias (Leeb et al., 2003).

O grupo de autocuidado teve duração média de 1 hora, onde foram abordados temas como: a importância do uso contínuo de medicações, esclarecimento sobre a doença, como proteger as articulações durante a realização das atividades do dia a dia com base nos princípios de proteção articular e conservação de energia e o uso de tecnologia assistiva. Inicialmente foi realizada uma apresentação expositiva com auxílio de slides, por meio de linguagens simples e ilustrações, seguida de espaço para trocas de experiência entre os pacientes e dúvidas com a equipe. O grupo dialógico entre os discentes dos dois cursos aconteceu com duração de 1 hora, no qual foi apresentado de forma expositiva e discutido entre os participantes a abordagem da Terapia Ocupacional com este público.

O acompanhamento individual consistiu em 3 encontros, nos quais foram realizadas indicações, confecções e a disponibilização de dispositivos de Tecnologia Assistiva (TA) para a paciente, assim como realizado o treino das atividades de vida diária com prejuízos identificados na avaliação. Foi possível atender as demandas específicas da paciente, com relação à disponibilização de dispositivos de TA por meio da interface entre o projeto de extensão "Reabilitação Funcional Musculoesquelética pós-Febre Chikungunya" e a pesquisa intitulada "Atenção à Saúde de Pessoas com Doenças Reumatológicas: Desenvolvimento de Produtos Assistivos e Formação de Recursos Humanos em Tecnologia Assistiva", aprovada na Chamada Pública FACEPE 10/2017, Programa Pesquisa para o sus: Gestão Compartilhada em Saúde/PPsus-Pernambuco, CNPq/MS/sES/FACEPE. Visto que a paciente apresentava o perfil e critérios de elegibilidade, foi possível incluí-la no projeto e fornecer recursos de TA indicados. O projeto de pesquisa obteve a aprovação do comitê do Centro de Ciências da saúde da UFPE SOb o registro dO CAAE: 71269417.0.0000.5208, a partir da resolução 466/2012 do Conselho Nacional de Saúde. Para todos os pacientes, os procedimentos de sigilo e confidencialidade foram preservados, assim como foram autorizados o uso de dados e imagens.

\section{Resultados}

A partir da participação no projeto de extensão com pacientes pós Febre Chikungunya, foram realizadas pela Terapia Ocupacional as seguintes ações: avaliação terapêutica ocupacional, discussão em equipe, realização de dois grupos de autocuidado e de um grupo dialógico de discentes da Fisioterapia e Terapia Ocupacional, e acompanhamento individualizado de uma das pacientes participantes do mesmo projeto.

A atuação da Terapia Ocupacional envolveu estratégias de educação em saúde, indicação de recursos de 
Tecnologia Assistiva e treino de AVD, além de orientações para o modo adequado de realizações de atividades durante todo o processo de intervenção. Para exposição dos achados no presente estudo, as ações desenvolvidas foram então categorizadas de acordo com o foco das intervenções, como seguem.

\section{Avaliação terapêutica ocupacional}

Participaram do estudo 10 pessoas com diagnóstico de Febre Chikungunya, sendo 8 do sexo feminino e 2 do sexo masculino, com idade entre 48 e 74 anos. Apenas 8 participantes responderam ao instrumento COPM, pois 2 pessoas não compareceram ao momento de avaliação. Destes, 1 paciente foi indicada para o atendimento terapêutico ocupacional individualizado em decorrência das limitações dos membros superiores que repercutiam diretamente em suas AVD, AIVD e trabalho de forma significativa.

A partir da COPM, observou-se que todos os participantes apresentavam algum comprometimento em seu desempenho ocupacional (Tabela 1). As limitações variaram entre as atividades de autocuidado, produtividade e lazer. Destas, destacam-se: vestir/despir, calçar sapatos, higienização no vaso, lavar pratos, passar roupas, varrer a casa, cozinhar, fazer transferências, subir em ônibus, escrever. Os participantes referiram que as dificuldades em seu desempenho eram decorrentes dos sintomas pós-Febre Chikungunya, como dores nas articulações, mialgia, edema e rigidez matinal principalmente nos joelhos, quadril e nas mãos, que persistiam mesmo após 2 anos de diagnóstico.

TABELA 1 - RESULTADOS DAS AVALIAÇÕES INICIAIS COM COPM. RECIFE-PE, 2018

\begin{tabular}{|c|c|c|c|}
\hline Pacientes & Queixas gerais & Desempenho & Satisfação \\
\hline $\mathrm{P} 1$ & $\begin{array}{l}\text { Banho (lavar parte posterior) }{ }^{1} \text {, abrir potes }{ }^{1} \text {, cozinhar (des- } \\
\text { cascar alimentos) }{ }^{1} \text {, varrer }{ }^{1} \text {, usar tesoura }{ }^{1} \text {, higiene (uso do } \\
\text { vaso), vestuário, alimentação (uso de faca), transferências, } \\
\text { estender roupas, lavar pratos, escrever }\end{array}$ & 2,4 & 1,0 \\
\hline P2 & Arrumar a casa & 5,0 & 7,0 \\
\hline P3 & Transferências, mobilidade & 2,0 & 1,0 \\
\hline P4 & Vestir e calçar, mobilidade, subir no ônibus & 3,6 & 3,0 \\
\hline P5 & Varrer, lavar pratos, estender roupas, cozinhar & 3,2 & 3,0 \\
\hline P6 & Abotoar, pegar moeda & 3,0 & 10,0 \\
\hline P7 & $\begin{array}{l}\text { Pentear cabelo }{ }^{2} \text {, subir escadas }{ }^{2} \text {, lavar panelas², passar rou- } \\
\text { pas }^{2} \text {, lavar roupas }{ }^{2} \text {, vestir, usar faca }\end{array}$ & 5,6 & 2,0 \\
\hline P8 & Vestir, subir no ônibus, varrer, lavar banheiro & 1,5 & 4,0 \\
\hline
\end{tabular}

${ }^{\top}$ Atividades problemas mais importantes, segundo a paciente P1.

${ }^{2}$ Atividades problemas mais importantes, segundo a paciente $P 7$.

Fonte: Elaboração própria, 2018.

Para os pacientes que apresentaram maiores limitações e/ou queixas no(s) membro(s) superior(es) foi aplicado o instrumento SACRAH, totalizando 2 casos. Nesta avaliação (Tabela 2), constatou-se que as 2 participantes apresentaram dor máxima frequentemente durante o trabalho intenso e pontuação acima de 6 no que diz respeito a rigidez matinal. Em relação às tarefas com maior dificuldade, com variação entre 8 e 10, destacaram-se afivelar um cinto, fazer as unhas, girar a maçaneta de uma porta ou janela, girar uma chave comum na fechadura, passar as folhas de um jornal e cortar com uma faca de cozinha. 
TABELA 2 - ESCORES DAS AVALIAÇÕES INICIAIS COM O SACRAH. RECIFE-PE, 2018.

\begin{tabular}{|c|c|c|}
\hline Atividades & Escore P1 & Escore P7 \\
\hline \multicolumn{3}{|l|}{ Função da mão } \\
\hline Travar/destravar uma porta & 5 & 3 \\
\hline Girar a maçaneta de uma porta ou janela & 9 & 2 \\
\hline Atacar um sutiã/dar nó em gravata & 9 & 2 \\
\hline Abotoar/desabotoar uma camisa ou blusa & 9 & 2 \\
\hline Girar uma torneira & 8 & 3 \\
\hline Abrir/fechar um zíper & 7 & 1 \\
\hline Amarrar cadarços & 8 & 2 \\
\hline Desenroscar a tampa de um tubo de pasta de dentes & 8 & 2 \\
\hline Acender um fósforo & 8 & 3 \\
\hline Segurar uma caneta & 9 & 2 \\
\hline Afivelar um cinto & 8 & 2 \\
\hline Fazer as unhas & 10 & 4 \\
\hline Girar uma chave comum na fechadura & 7 & 2 \\
\hline Passar as folhas de um jornal & 8 & 2 \\
\hline Manusear cédulas & 8 & 3 \\
\hline Escrever & 9 & 3 \\
\hline Cortar com uma faca de cozinha & 10 & 2 \\
\hline Média $1(M 1)$ & 8,23 & 2,35 \\
\hline \multicolumn{3}{|l|}{ Rigidez } \\
\hline Rigidez matinal & 9 & 8 \\
\hline Rigidez no decorrer do dia & 5 & 2 \\
\hline Média 2 (M2) & 7 & 5 \\
\hline \multicolumn{3}{|l|}{ Dor } \\
\hline Durante trabalho diário regular & 10 & 8 \\
\hline Durante trabalho intenso & 10 & 10 \\
\hline Em momentos de inatividade & 7 & 4 \\
\hline Durante a noite & 10 & 8 \\
\hline Média 3 (M3) & 9,25 & 7,5 \\
\hline SACRAH $(M 1+M 2+M 3) / 3$ & 8,16 & 4,95 \\
\hline
\end{tabular}

A identificação da demanda para acompanhamento individualizado foi efetivada a partir da observação dos resultados das avaliações iniciais dos pacientes, na qual uma participante se destacou por apresentar grande limitações relativas ao desempenho e satisfação de 5 ou mais atividades apontadas como problemas; pontuação do SACRAH acima de 5,O; afastamento de atividades significativas e laborais, além das queixas 
de dores. Deste modo, a paciente $\mathrm{P} 1$ apresentou elegibilidade para a assistência individualizada. Os demais pacientes foram então contemplados com orientações e recebimento de cartilha em momento de grupo.

Após discussão em equipe, as intervenções da Terapia Ocupacional foram subsidiadas pela literatura clínica pertinente acerca do vírus Chikungunya, correlacionados com o respaldo teórico e metodológico do campo da Terapia Ocupacional na Reumatologia.

\section{Grupo de Educação em Saúde pós-Febre Chikungunya}

Estiveram presentes no grupo de educação em saúde 10 indivíduos acometidos pós-Febre Chikungunya. $\mathrm{O}$ grupo, conduzido pela Terapeuta Ocupacional e pelos discentes do mesmo curso, abordou, inicialmente, a importância de conhecer a doença, seus sintomas, possíveis manejos no tratamento, a importância do uso contínuo de medicações e quais as medidas preventivas contra os focos dos mosquitos transmissores, empoderando os indivíduos. Entende-se que, na condução de uma intervenção em grupo, faz-se necessário que o terapeuta esteja atento às demandas que surgem no decorrer da discussão, sendo capaz de identificá-las e acolhê-las. Assim como abordar a temática de forma contextualizada, com sentido e significado aos participantes, de modo a estimular os mesmos a engajarem-se na proposta de construção conjunta de conhecimento.

A partir dos sintomas mais comuns apresentados pelos participantes foi possível ouvir e perceber a dificuldade enfrentada desde o surgimento da doença, principalmente nas atividades básicas como vestir e despir. A condição crônica também refletia no contexto emocional e social, visto que muitos relataram as limitações para sair de casa, utilizar transportes e participar de atividades de lazer. Diante dos relatos de cada participante, foi possível observar que o grupo proporcionou o momento para compartilhar sentimentos, trocar informações e promover um enfrentamento da doença de forma coletiva.

Frente às dificuldades expostas, foram repassadas orientações sobre o respeito à dor e os limites do corpo, redução da força e do esforço para a realização de determinadas atividades cotidianas, distribuição da carga em mais de uma articulação optando por articulações maiores e mais fortes, evitar permanecer na mesma posição por tempo prolongado, pausa durante atividades que exigem maior esforço, segurar e manusear objetos de forma adequada. Todas as orientações basearam-se nos princípios de proteção articular e conservação de energia, os quais objetivam a redução da dor, fadiga e desgaste articular, a fim de facilitar a realização das AVD e AIVD de modo mais seguro.

As orientações envolveram a indicação de formas apropriadas para realização de atividades como varrer a casa, lavar pratos, torcer panos, abrir potes, torneiras e maçanetas, estender roupas no varal, segurar e carregar objetos pesados. Também foram apresentados exemplos de recursos de Tecnologia Assistiva como escova para banho com cabo alongado, engrossadores para vassoura e esponja de lavar pratos com velcro. Tais dispositivos de assistência são benéficos no auxílio à realização das atividades e amenizam os impactos causados nas articulações. Ao final do grupo foram entregues cartilhas de orientações a cada participante, como complemento das reflexões.

\section{Atendimento terapêutico ocupacional individualizado}

A paciente $\mathrm{P}_{1}, 66$ anos de idade, aposentada, atualmente trabalha como recepcionista em uma clínica para idosos. Associado ao quadro de Febre Chikungunya, apresenta rizartrose bilateral, cirurgias no ombro, túnel do carpo com a realização de 2 cirurgias, além do $3^{\circ}$ e $4^{\circ}$ dedo em gatilho na mão esquerda. O quadro de rizartrose da paciente em questão foi agravado após seu acometimento da Febre Chikungunya, corroborando com os achados na literatura que expõem o aparecimento ou agravo de condições reumatológicas prévias após caso de FC, sendo esta uma realidade da amostra do presente estudo.

No primeiro encontro, a partir da COPM, foram identificadas as 5 atividades mais importantes que a paciente apresentava dificuldades, entre elas o banho (lavar a região posterior), abrir potes, descascar alimentos, varrer a casa e usar tesouras, com média de desempenho de 2.4 e satisfação 5. Entretanto, além dessas relatou dificuldades significativas em outras tarefas como lavar pratos, cortar carnes e legumes, segurar copos e escrever, sendo esta última bastante utilizada em seu trabaIho de recepcionista. 
No segundo encontro foram realizados treinos das seguintes atividades significativas para a paciente com o uso dos dispositivos de Tecnologia Assistiva e com adequação da postura para um menor esforço (Figura 1, Figura 2, Tabela 3): uso de copo com alças (Figura 2, item D); uso de vassoura com adaptação para preensão (Figura 2, item E); descascador de alimentos (Figura 2, item G); abrir tampa de garrafa utilizando dispositivo (Figura 2, item $\mathrm{H}$ ); cortar alimento utilizando faca com engrossador (Figura 2, item I) e tábua adaptada com espetos (Figura 2, item J); lavagem de corpo durante banho com uso de escovão com cabo adaptado (Figura 2, item K), e escrita com lápis com engrossador (Figura 2, item L). Foi possível perceber e ouvir da paciente uma melhora importante durante o treinamento com e sem uso dos dispositivos. As orientações de proteção articular e conservação de energia foram reforçadas durante a realização das atividades para que a paciente pudesse perceber em prática a diferença entre a forma apropriada e a forma incorreta de realização.

No último encontro, que aconteceu um mês após entrega dos dispositivos, a paciente relatou estar desempenhando suas atividades com redução das dores nas articulações e maior independência. De acordo com os escores de reavaliação do instrumento SACRAH, houve ganhos na pontuação média a qual mudou de 6,73 para 5,19 . Na COPM, sua média de desempenho subiu de 2.4 para 5, enquanto a média de satisfação foi elevada de 5 para 7 após uso dos dispositivos.

\section{Grupo dialógico dos discentes de Fisioterapia e Terapia Ocupacional}

Após o desenvolvimento das ações de intervenções em grupo e individualizadas, foi realizado um grupo dialógico a fim de compartilhar os principais resultados a partir do olhar da terapia ocupacional, conduzido pelos alunos do mesmo curso. Compareceram no grupo a docente coordenadora e todos os discentes de Fisioterapia que participaram do projeto de extensão "Reabilitação Funcional Musculoesquelética pós-Febre Chikungunya", os quais tiveram a oportunidade de contribuir para a discussão em conjunto acerca do caso clínico apresentado.

FIGURA 1 ÓRTESE PARA RIZARTROSE INDICADA PARA PACIENTE COM QUADRO DE FEBRE CHIKUNGUNYA. RECIFE-PE, 2018

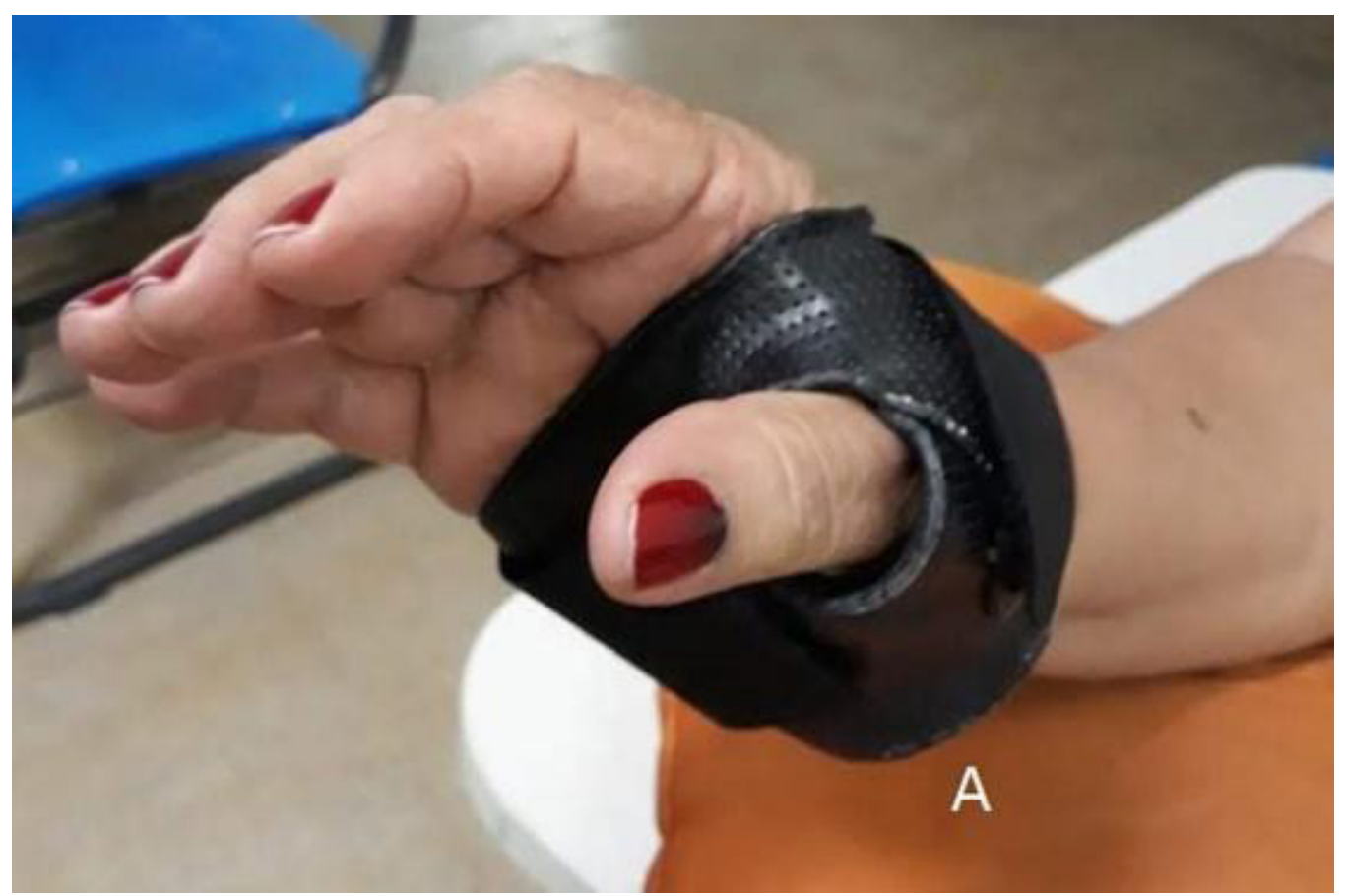


FIGURA 2 DISPOSITIVOS DE TECNOLOGIA ASSISTIVA INDICADOS PARA PACIENTE COM QUADRO DE FEBRE CHIKUNGUNYA

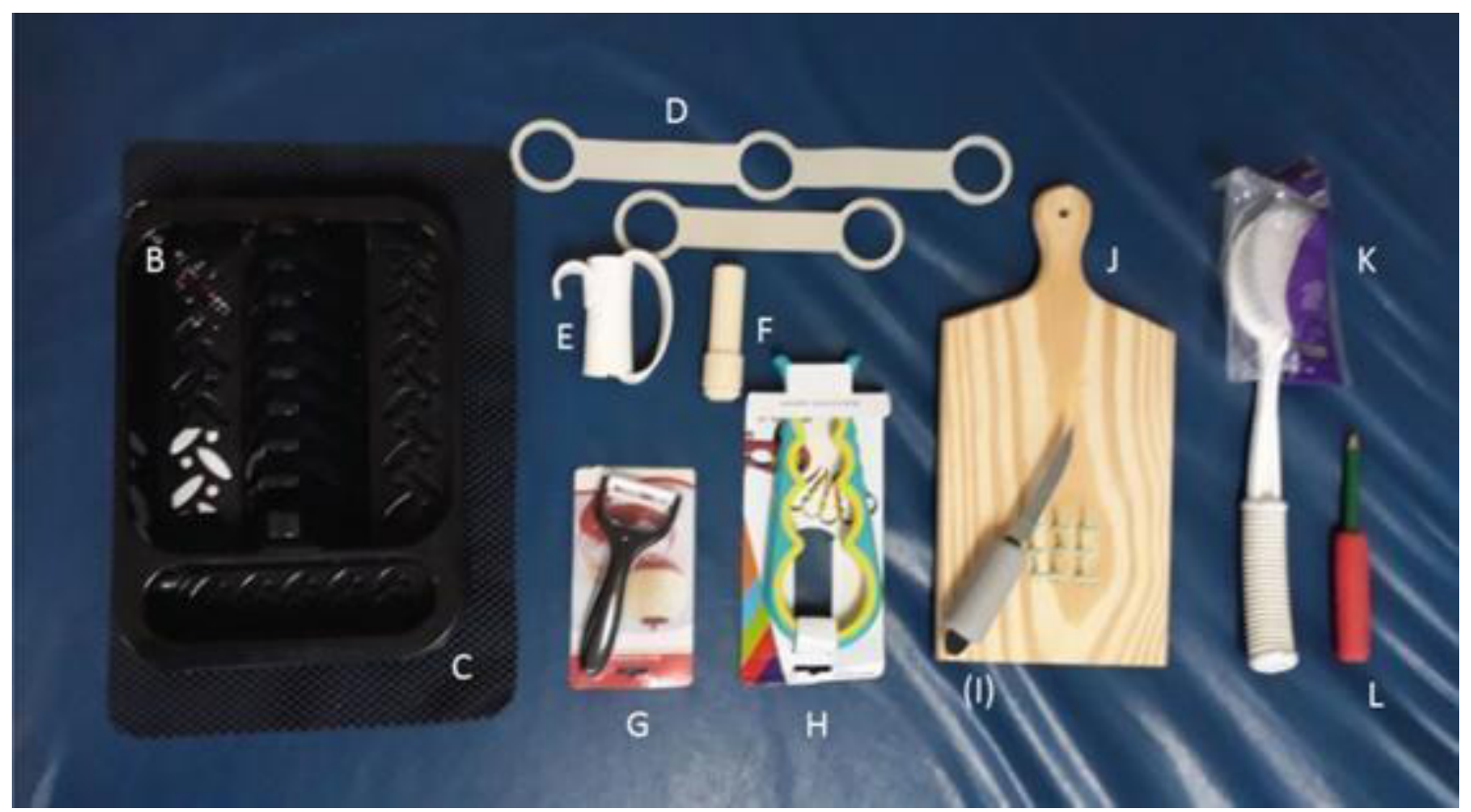

Através de uma apresentação expositiva em slides elaborada pela docente e discentes em terapia ocupacional, foi exposto o histórico da paciente selecionada para o atendimento individualizado com foco nas áreas de ocupações comprometidas. Em seguida foram apresentados os estudos que embasaram as intervenções da Terapia Ocupacional, os instrumentos utilizados durante avaliação e seus resultados.

No decorrer do grupo foi possível discutir a importância da integração de profissionais da área de saúde, pois através da troca de saberes ampliam-se as possibilidades do cuidado e atenção com o indivíduo, sem descaracterizar a individualidade de cada profissão. A abordagem interdisciplinar pode ser benéfica à população acometida pela Febre Chikungunya pela complexidade dos casos, visto que cada profissional pode contribuir de acordo com a especificidade de sua expertise, favorecendo melhoria na qualidade de vida da população.

\section{Discussão}

Os participantes do presente estudo mostraram dificuldades no desempenho funcional em diferentes áreas de ocupação, como as atividades de vida diária, atividades instrumentais de vida diária, atividades laborais, de produtividade, de lazer e participação social. Os achados a partir da avaliação inicial apontaram que os comprometimentos advindos do acometimento pós-Febre Chikungunya implicaram em agravos nos quadros clínicos de doenças reumatológicas previamente instaladas, assim como o aparecimento de novas limitações funcionais. As sequelas da infecção pelo vírus Chikungunya vem interferindo diretamente na qualidade de vida dos indivíduos, implicando em limitações funcionais importantes no público acometido, destacando a artralgia como uma das principais queixas frente à possibilidade de cronificação. A alteração da mobilidade, as doenças reumatológicas e as questões emocionais como depressão e baixa de autoestima, também são citadas como desafios para a clientela (Azevedo, Oliveira e Vasconcelos, 2015; Castro, Lima e Nascimento, 2016; Marques et al., 2017; Viana et al., 2018; Sales et al., 2018).

Os achados do presente estudo corroboram com o que Fulfaro et al. (2012), Parreira et al. (2013) e Almeida et al. (2015) apontam quanto ao declínio funcional causado pelo quadro clínico de doenças reumatológicas. Este 
TABELA 3 RECURSOS DE TECNOLOGIAS ASSISTIVAS INDICADAS DE ACORDO COM AS DEMANDAS DA PACIENTE COM QUADRO DE FEBRE CHIKUNGUNYA E FUNÇÕES. RECIFE-PE, 2018

\section{RECURSOS DE TECNOLOGIAS ASSISTIVAS*}

Órtese para rizartrose $(\mathrm{A})$

Escorredor de pratos (B)

Antiderrapante (C)

Adaptações para copo (D)

Adaptação para vassoura (E)

Adaptador universal (F) de plástico $(\mathrm{ABS})^{* *}$

Descascador (G)

Abridor de potes $(\mathrm{H})$

Adaptação para faca (I)

Tábua adaptada (J)

Adaptação para banho (K)

Adaptação para escrita (L)

\section{FUNÇÃO}

Estabilizar a articulação trapézio-metacarpiana, visando diminuição da dor e desgaste articular durante realização das atividades. O material utilizado foi o termoplástico de baixa temperatura.

Prevenir o desgaste articular dos polegares durante manipulação dos pratos na lavagem, o escorredor serve para apoiar os pratos dentro da pia enquanto passa sabão, esponja e água.

Apoiar o escorredor (B) durante a passagem de sabão no prato, não sendo necessário segurá-lo durante o procedimento desta etapa.

Favorecer a preensão cilíndrica, reduzir a fadiga e o desgaste das articulações das mãos.

Direcionar o uso da preensão palmar para reduzir o uso da força ao segurar e movimentar a vassoura.

Favorecer a preensão e menor desgaste articular de mãos, a partir de diâmetro regulável para encaixe de lápis, canetas e agulhas de crochê.

Favorecer a preensão palmar e reduzir o esforço das articulações menores da mão ao substituir o uso da faca.

Facilitar abertura de diferentes tamanhos de tampas, favorece preensão em gancho, protegendo as articulações menores e minimizando as dores durante o movimento para abrir.

Aumentar o diâmetro da preensão em mão fechada, reduzindo a fadiga e amenizando a força necessária para cortar os alimentos.

Segurar os alimentos durante o corte, prevenindo esforço das articulações da mão durante preensão do alimento.

Favorecer maior alcance durante a lavagem do corpo, evitando a fadiga dos membros superiores para alcançar as costas e a inclinação, além da sobrecarga na coluna para lavagem das pernas.

Favorecer a pinça trípode, minimizar a força e desgaste articular de mão durante escrita.

*(A) Dispositivo confeccionado pela terapeuta ocupacional da equipe. (B); (C); (G); (H) Dispositivos encontrados no mercado. (D); (I); (L)Dispositivos da empresa Mercur. (E) Dispositivo da empresa Autonomie. (F)Adaptador universal (patente BR 102018068307 1). (J); (K) Dispositivos adaptados pela equipe.

**ABS - Acrilonitrila Butadieno Estireno.

Fonte: Elaboração própria, 2018. 
declínio foi identificado progressivamente pela perda da capacidade de executar tanto atividades de vida diária quanto atividades instrumentais da vida diária, assim como os pacientes assistidos pelo estudo apresentaram limitações nestas áreas ocupacionais. Nessa perspectiva, Hammar e Hakansson (2013) demonstraram, em seu estudo exploratório com mulheres com condições reumatológicas, que tais comprometimentos prejudicam a realização de atividades produtivas e laborais, no que se diz respeito ao trabalho, havendo a possibilidade de evasão da profissão devido ao alto nível e intensidade da dor, apresentando baixo desempenho no exercício da ocupação.

Nesse sentido, com respaldo nos princípios e intervenções da terapia ocupacional na reumatologia, as ações realizadas no presente estudo foram de acordo com as possibilidades de atuação evidenciadas em estudos clínicos, tais como orientações e educação do paciente acerca da doença numa abordagem individual ou em grupo; estímulo à mudança de hábitos; práticas fundamentadas nos princípios de proteção articular e conservação de energia; modificação de atividades e ambientes; indicação, confecção e treino do uso de dispositivos de tecnologia assistiva e adaptações (Boer et al., 2009; Hammar e Hakansson, 2013; Almeida et al., 2015; Paula, 2017; Amaral et al., 2018; Santos et al., 2018). Tais estratégias corroboram com as evidências científicas de outros autores da literatura nacional e internacional, que reuniram, por meio de revisões, pesquisas baseadas em evidência científica no âmbito da terapia ocupacional e reumatologia (Miranda, 2012; Hand, Law e Mccoll, 2011; Aebischer, Elsig e Taeymans, 2016).

A amostra deste estudo está dentro da faixa etária de 48 a 74 anos de idade, tendo o sexo feminino como predominante entre os participantes. Quanto aos fatores de risco para o acometimento da Febre Chikungunya, Azevedo, Oliveira e Vasconcelos (2015) sugeriram em seu estudo a idade como um destes fatores, relacionado à continuidade da artralgia e artrite em pacientes pós-Febre Chikungunya. Nesse segmento, Marques et al. (2017) apontam outros fatores de risco associados com a cronificação e piora do prognóstico da doença, tais como o sexo feminino, faixa etária maior que 40 anos de idade, presença de edema, rigidez articular, poliartrite, diagnóstico prévio de doença articular, além de comorbidades, a citar a diabetes mellitus.
A intervenção em abordagem grupal, realizado pela docente e alunos do curso de Terapia Ocupacional, respeitou a individualidade de cada paciente, abordando de forma clara e contextualizada o quadro pós-Febre Chikungunya, discutindo estratégias e ampliando o leque de possibilidades de enfrentamento das situações limitantes nas ocupações dos indivíduos na esfera biopsicossocial. A estratégia da intervenção grupal associada à educação em saúde também foi utilizada no ensaio clínico randomizado de Amaral et al. (2018) por meio de grupos de intervenção e controle. As autoras evidenciaram que os grupos, compostos por pacientes mulheres diagnosticadas com doença reumatológica, mostrou-se relevante para os participantes, visto o expressivo interesse e comprometimento dos mesmos, no que se refere a completa adesão aos encontros.

Nesse sentido, na presente experiência foi possível observar discursos dos participantes ao final da vivência, enquanto relatos positivos de sentir-se contemplados pelo momento grupal, atribuindo ao grupo o rico potencial para troca de conhecimento e experiência entre indivíduos com demandas em comum. $\mathrm{O}$ uso da estratégia da educação em saúde nos atendimentos em grupo almeja o melhor conhecimento da doença por parte dos participantes, empoderando o sujeito enquanto protagonista no processo saúde-doença, além de estimulá-los a participaram ativamente de reflexões conjuntas acerca do tema (Toquetti et al., 2008; Noordhoek e Loschiavo, 2005; Amaral et al. 2018). Por se tratar de uma população em situação de pós-acometimento de uma arbovirose, com sequelas principalmente reumatológicas, a importância da atuação do terapeuta ocupacional na abordagem grupal é válida ao proporcionar, entre os indivíduos, o desenvolvimento do vínculo, autonomia e empoderamento quanto sua atual condição de saúde.

A partir do encontro em grupo, pôde-se estimular a criação de um ambiente propício à troca de saberes, possibilitando uma reflexão conjunta acerca das consequências da doença na vida dos indivíduos. Deste modo, as estratégias utilizadas no presente estudo, no momento de intervenção em grupo, vão de acordo com os pressupostos apontados por Bianchin et al. (2010) e Fulfaro et al. (2012), os quais indicam a adequação do objetivo terapêutico a partir da demanda da clientela, lançando mão de orientações e estímulos à mudança de hábitos, ambiente ou modo de realização de atividades que 
estejam prejudicando o desempenho ocupacional eficaz dos pacientes em suas atividades.

Os momentos em grupo proporcionaram aos participantes o estímulo à interação social, partilha de conhecimentos e respeito mútuo às experiências adquiridas pelo próximo, assim como argumentam Noordhoek e Loschiano (2005). Segundo as autoras, a abordagem grupal é indicada para pacientes acometidos de doenças crônicas, visto o potencial dos grupos na educação em saúde, no conhecimento e empoderamento da doença, estímulo à postura e participação ativa dos indivíduos frente às diferentes condições de saúde.

Uma vez identificadas as demandas da população quanto às queixas do modo de realização de atividades, fez-se necessário abordar estratégias dos modos adequados de feitura destas. Uma maneira para alcançar tal solução foi a confecção e distribuição de uma cartilha de orientação, abordando princípios de proteção articular e conservação de energia. De acordo com Torquetti et al. (2008) e Fulfaro et al. (2012), estes princípios são comumente temas de intervenções em grupo, pois objetivam a redução da dor, estresse articular e prevenção de deformidades a partir de mudanças de hábitos, adaptações em materiais de uso diário e adequações posturais favorecendo melhor realização de atividades com conforto e segurança. Para o presente estudo, as orientações e estratégias utilizadas baseadas nestes princípios foram contextualizadas para os pacientes assistidos pela Terapia Ocupacional, ao levar em consideração suas histórias, seus desejos, queixas e potencialidades, almejando uma realização mais autônoma das atividades cotidianas comprometidas relatadas.

A intervenção e acompanhamento individualizado da paciente pela Terapia Ocupacional destacaram a abordagem dos princípios da proteção articular e conservação de energia quando utilizadas para manutenção da dor e fadiga. Para tal, o terapeuta ocupacional utilizando-se dos princípios ergonômicos e biomecânicos está apto a proporcionar uma melhor forma de realização das atividades e ocupações significativas, a fim de evitar estresse articular e prevenir agravos, causando impactos benéficos na realização de modo adequado de ocupações e atividades cotidianas (Hand, Law e Mccoll, 2011; Almeida et al., 2015; Paula, 2017).

As intervenções incluíram a utilização da tecnologia assistiva e dos dispositivos de assistência, que, corroborando com as evidências encontradas nos estudos de Paula (2017), Silva e Massa (2015), Veehof et al. (2006), Boer et al. (2009) e Amaral et al. (2018), estes servem como auxílio na realização das atividades, diminuem a dor e a força sobre articulações. Após a avaliação, prescrição e treino, a paciente acompanhada neste estudo recebeu dispositivos de assistência para as atividades de alimentação e preparo de alimentos, escrita, banho, varrer casa e lavar pratos. Em reavaliação após um mês de uso dos dispositivos, a paciente relatou melhorias nas realizações das ocupações destacadas como significativas para ela, com menores ou ausências de queixas de dores ou incômodos em articulações distais. A partir dos resultados das avaliações aplicadas, a paciente acompanhada individualmente apresentou, em geral, maiores dificuldades nas atividades de autocuidado e de produtividade, sendo contemplada com dispositivos de assistência para cada atividade em questão. Os dispositivos de assistência são bem aceitos por pacientes com doenças reumatológicas como foi evidenciado no estudo de Santos et al. (2018), onde a maioria dos entrevistados usava com frequência os dispositivos de assistência em sua rotina e indicaria o uso destes para outros indivíduos com a mesma dificuldade.

\section{CONCLUSÃO}

A partir do presente estudo, identificou-se que as intervenções realizadas com o público pós-Febre Chikungunya mostraram-se importantes para a construção do conhecimento sobre a doença e melhor enfrentamento da mesma a partir do foco da Terapia Ocupacional. As intervenções terapêuticas ocupacionais apresentaram um leque ampliado de possibilidades de assistência, desde a abordagem em grupo até a individual, tendo os princípios de reumatologia, proteção articular e conservação de energia como importantes referenciais para o subsídio de ações terapêuticas, contemplando a utilização de recursos de Tecnologia Assistiva, treinos e orientações para o melhor desempenho funcional de atividades e ocupações significativas. Nesse sentido, as orientações discutidas no momento em grupo foram contextualizadas e com significado para a população assistida. Cabe ressaltar que entre os recursos facilitadores do processo de acompanhamento estão as cartilhas de orientações que possibilitaram o alcance da informação para além do momento de intervenção. 
Observou-se ainda que a experiência demonstrou o potencial das ações da terapia ocupacional com grupos terapêuticos de educação em saúde, ao favorecer a criação de espaços para a reflexão conjunta de questões pertinentes para a saúde da população acometida pós-Febre Chikungunya, assim como no atendimento individualizado desta população. Considerando o treino de atividades significativas, desenvolvimento ou recuperação de habilidades, o controle de sintomas, empoderamento do indivíduo frente ao caso clínico e orientações como mediadoras deste processo, subsidiadas pela escuta e construção compartilhada do conhecimento, estimulou-se a reflexão sobre temáticas significativas para os participantes, fazendo-se necessária a criação de estratégias de melhorias para o bem-estar biopsicossocial do público.

Para a redução dos impactos nas articulações, diminuição das dores e melhora no desempenho durante a execução de atividades, são recomendados os treinos das atividades de vida diária e atividades instrumentais de vida diária com o uso dos dispositivos de Tecnologia Assistiva, apontados neste estudo como importantes ferramentas para o planejamento e desenvolvimento dos objetivos terapêuticos. O uso da TA nas intervenções terapêuticas ocupacionais evidenciou o potencial dos recursos de assistência na melhoria do desempenho funcional em ocupações de interesse para o indivíduo.

Desta feita, espera-se que o estudo contribua com a literatura científica para posteriores pesquisas na temática da assistência da terapia ocupacional com o público pós-Febre Chikungunya.

\section{AgradeCimentos}

Agradecemos ao Decit/sctie/MS, CNPq, SES/PE e fACEPE pela colaboração neste estudo.

\section{ReFERENCIAS BIBLIOGRÁFICAS}

Aebischer, B., Elsig, S. \& Taeymans, J. (2016). Effectiveness of physical and occupational therapy on pain, function and quality of life in patients with trapeziometacarpal osteoarthritis-A systematic review and meta-analysis. Hand Therapy, 21(1), 5-15. https://doi. org/10.1177/1758998315614037

Aguiar, B. S., Lorenz, C., Virginio, F., Suesdek, L. \& Chiaravalloti-Neto, F. (2018). Potential risks of Zika and chikungunya outbreaks in Brazil:
A modeling study. International Society for Infectious Diseases, 70, 9-20. https://doi.org/10.1016/j.ijid.2018.02.007

Almeida, P.H.T.Q., Pontes, T. B., Matheus, J. P. C., Muniz, L. F. \& Mota, L. M. H. (2015). Terapia ocupacional na artrite reumatoide: o que o reumatologista precisa saber? Revista Brasileira de Reumatologia, 55(3), 272-280. https://doi.org/10.1016/j.rbr.2014.07.008

Amaral, D. S., Duarte, A. L. B. P, Barros, S. S., Cavalcanti, S. V., Ranzolin, A., Leite, V. M. M., Dantas, A. T., Oliveira, A. S. C. R. C., Santos, P. S., Silva, J. C. A \& Marques, C. D. L. (2018). Assistive devices: na effective strategy in non-pharmacological treatment for hand osteoarthritis - randomized clinical trial. Rheumatology International, 38, 343-351. https://doi.org/10.1007/s00296-017-3892-1

Azevedo, R. S. S., Oliveira, C. S. \& Vasconcelos, P. F. C. (2015). Risco do chikungunya para o Brasil. Revista de Saúde Pública, 49, 1-6. https://doi.org/10.1590/S0034-8910.2015049006219

Bianchin, M. A., Paula, G. A. S., Carvalho, M. P., Acayaba, R. \& Chueire, R. (2010). Manual de orientações de terapia ocupacional quanto à proteção articular para pacientes com artrite reumatoide. Medicina de Reabilitação, 29(1), 8-23. http://files.bvs.br/upload/S/0103-5894/2010/v29n1/a006.pdf

Boer, I. G., Peeters, A. J., Ronday, H. K., Mertens, B. J. A., Huizinga, T. W. J. \& Vliet Vlieland, T. P. M. (2009). Assistive devices: usage in patients with rheumatoid arthritis. Clinical Rheumatology, 28, 119-128. https://doi.org/10.1007/s10067-008-0989-7

Castro, A. P. C. R., Lima, R. A. \& Nascimento, J. S. (2016). Chikungunya: vision of the pain clinician. Revista Dor, 17(4), 299-302. http://www. scielo.br/pdf/rdor/v17n4/pt_1806-0013-rdor-17-04-0299.pdf

Donalisio, M. R. \& Freitas, A. R. R. (2015). Chikungunya no Brasil: um desafio emergente. Revista Brasileira de Epidemiologia, 18(1), 5-283. https://doi.org/10.1590/1980-5497201500010022

Ferreira, V. C. B. \& Marques, C. D. L. (2008). Avaliação e quantificação de afecções reumáticas crônicas das mãos através do questionário Sacrah. Jornal da Lirnne, 4(1), 171-177

Fulfaro, M. A., Zamper, S. S. S., Luzo, M. C. M. \& Almeida, M. H. M. (2012). Caracterização funcional de idosos com artrite reumatóide. Estudos Interdisciplinares sobre o Envelhecimento, 17(2), 305-319. https://seer.ufrgs.br/RevEnvelhecer/article/view/20210/25383

Hammar, I. O. \& Hakansson, C. (2013). The importance for daily occupations of perceiving good health: Perceptions among women with rheumatic diseases. Scandinavian Journal of Occupational Therapy, 20, 92-92. https://doi.org/10.3109/11038128.2012.699978

Hand, C., Law, M. \& Mccoll, M. A. (2011). Occupational Therapy Interventions for Chronic Diseases: A Scoping Review. The American Journal of Occupational Therapy, 65(4), 428-436. https://doi. org/10.5014/ajot.2011.002071

Law, M., Baptiste, S., Carswell, A., McColl, M. A., Polatajko, H., \& Pollock, N. (2009). Medida Canadense de Desempenho Ocupacional (COPM) (Lívia de Castro Magalhães, Lílian Vieira Magalhães \& Ana Amélia Cardoso, trad.). Belo Horizonte: Editora UFMG 
Leeb, B. F., Sautner, J., Andel, I. \& Rintelen, B. (2003). SACRAH: a score for assessment and quantification of chronic rheumatic affections of the hands. Rheumatology, 42(10), 8-1173. https:// doi.org/10.1093/rheumatology/keg319

Lima-Camara, T. N. (2016). Arboviroses emergentes e novos desafios para a saúde pública no Brasil. Revista de Saúde Pública, 50(36), 1-7. http://dx.doi.org/10.1590/S1518-8787.2016050006791

Marques, C. D. L., Duarte, A. L. B. P., Ranzolin, A., Dantas, A. T., Cavalcanti, N. G., Gonçalves, R. S. G., ... Teixeira, R. (2017). Recomendações da Sociedade Brasileira de Reumatologia para diagnóstico e tratamento da febre chikungunya. Parte 1-Diagnóstico e situações especiais. Revista Brasileira de Reumatologia, 57(2), 421-437. https://doi.org/10.1016/j.rbr.2017.05.004

Marques, C. D. L., Duarte, A. L. B. P., Ranzolin, A., Dantas, A. T., Cavalcanti, N. G., Gonçalves, R. S. G., ... Teixeira, R. (2017). Recomendações da Sociedade Brasileira de Reumatologia para diagnóstico e tratamento da febre chikungunya. Parte 2-Tratamento. Revista Brasileira de Reumatologia, 57(2), 438-451. https://doi.org/10.1016/j. rbr.2017.05.005

Miranda, L. C. G. (2012). Terapia ocupacional em reumatologia: prática baseada na evidência na artrite reumatoide [Dissertação de Especialização em Terapia Ocupacional, Instituto Politécnico do Porto]. Repositório Científico do Instituto Politécnico do Porto. http://recipp.ipp.pt/bitstream/10400.22/2310/1/PTE_LeonorMiranda_2012.pdf

Noordhoek, J. \& Loschiavo, F, Q. (2005). Intervenção da Terapia Ocupacional no Tratamento de Indivíduos com Doenças Reumáticas Utilizando a Abordagem da Proteção Articular. Revista Brasileira de Reumatologia, 45(4), 44-242. http://dx.doi.org/10.1590/ S0482-50042005000400008

Parreira, M. M., Cavalcanti, A., Cunha, J. H. da S., \& Cordeiro, J. J. R. (2013). Papéis ocupacionais de indivíduos em condições reumatológicas. Revista de Terapia Ocupacional da Universidade de São Paulo, 24(2), 33-127. https://doi.org/10.11606/issn.22386149.v24i2p127-133

Paula, P. M. S. (2017). Terapia Ocupacional e Tecnologia Assistiva: Funcionalidade para Pessoas com Artrite Reumatoide [Dissertação de Mestrado em Enfermagem, Faculdade de Medicina de São José do Rio Preto] Biblioteca Digital de Teses e Dissertações FAMERP. http://bdtd.famerp.br/handle/tede/381

Pineda, C., Muñoz-Louis, R., Caballero-Uribe, C. V. \& Viasus, D. (2016). Chikungunya in the region of the Americas. A challenge for rheumatologists and health care systems. Clinical Rheumatology, 35(10), 2381-2385. https://doi.org/10.1007/s10067-016-3390-y

Sales, G. M. P. G., Barbosa, I. C. P., Canejo Neta, L. M. S., Melo, P. L., Leitão, R. A. \& Melo, H. M. A. (2018). Treatment of chikungunya chronic arthritis: A systematic review. Revista da Associação Médica Brasileira, 64(1), 63-70. http://dx.doi.org/10.1590/18069282.64.01.63
Silva, N. M., Teixeira, R. A. G., Cardoso, C. G., Siqueira Junior, J. B., Coelho, G. E., \& Oliveira, E. S. F. (2018). Vigilância de chikungunya no Brasil: desafios no contexto da Saúde Pública. Epidemiologia e Serviços de Saúde, 27(3), 1-10. http://dx.doi.org/10.5123/s167949742018000300003

Santos, P. S., Martins, N. I. M., Leite, V. M. M., Sanguinetti, D. C. M., Paixão, L. K. P. A., Marques, C. D. L., \& Amaral, D. S. (2018). Uso de dispositivos de assistência por indivíduo com osteoartrite de mãos. Cadernos Brasileiros de Terapia Ocupacional, 26(1), 145-152. https://doi.org/10.4322/2526-8910.ctoAO0999

Silva, T. S. S. \& Massa, L. D. B. (2015). A utilização de órteses de membro superior em pacientes com artrite reumatoide: uma revisão de literatura no campo da terapia ocupacional. Cadernos Brasileiros de Terapia Ocupacional, 23(3), 647-659. https://doi. org/10.4322/0104-4931.ctoAR0522

Souza, C. A. F., Calixto, M. F., Marques, M. P., Oliveira, A. C. B. S. \& Alves, A. C. J. (2018). Uso de avaliação do desempenho para prescrição de dispositivos de tecnologia assistiva. Revista de Terapia Ocupacional da Universidade de São Paulo, 29(1), 34-40. https://doi.org/10.11606/issn.2238-6149.v29i1p34-40

Torquetti, A., Campos, T. da S., Noordhoek, J. \& Cassiano, J. G. (2008). Programas de proteção articular para indivíduos com artrite reumatoide: uma revisão da literatura. Revista de Terapia Ocupacional da Universidade de São Paulo, 19(2), 76-84. https://doi. org/10.11606/issn.2238-6149.v19i2p76-84

Veehof, M., Taal, E., Rasker, J., Lohmann, J., \& Van de Laar, M. (2006). Possession of assistive devices is related to improved psychological well-being in patients with rheumatic conditions. The Journal of Rheumatology, 33(8), 1679-1683. https://www.ncbi. nlm.nih.gov/pubmed/16881123

Viana, L. R. C., Pimenta, C. J. L., Araújo, E. M. N. F., Teófilo, T. J. S., Costa, T. F. \& Costa, K. N. F. M. (2018). Reemerging arboviruses: clinical-epidemiological profile of hospitalized elderly patients. Revista da Escola de Enfermagem da USP, 52, 1-7. https://doi.org/10.1590/ s1980-220x2017052103403 